\title{
TỔNG QUAN CẤY GHÉP IMPLANT NHA KHOA Có SỬ DỤNG MÁNG HƯỚNG DẪN PHẪU THUÂTT
}

\author{
Nguyễn Cao Thắng ${ }^{1}$, Trần Cao Bính ${ }^{2}$, \\ Đinh Diệu Hồng ${ }^{3}$, Đàm Văn Việt ${ }^{2}$
}

\section{TÓM TẮT}

Mục tiêu quan trọng để cấy ghép implant thành công là vị trí implant lý tưởng chính xác theo 3 chiều trong xương hàm, tồn tại lâu dài, đảm bảo được chực năng và thẩm mỹ. Việc sử dụng máng hướng dẫn phẫu thuật (MHDPT) trong cấy ghép implant được cho là mang lại nhiều lợi ích. Mục tiêu: Mô tả và phẩn tích gộp độ chính xác của vị trí implant được cấy ghép có sử dụng MHDPT, mức độ tiêu xương rìa, các biến chứng và tỷ lệ tồn tại của implant được cấy ghép có sử dụng MHDPT và không sử dụng MHDPT. Đối tượng và phương pháp nghiên cứu: Tổng quan hệ thổng và phân tích gộp dữ liệu về độ chính xác của vị trí implant, mức độ tiêu xương rìa, các biến chứng và tỷ lệ tồn tại của implant được cấy ghép có sử dụng MDHPT và không sử dụng MHDPT từ các bài báo, luận văn, luận án trên trang cơ sở dữ liệu Pubmed, Google Scholar, EBSCOhost Research Databases, thư viện trường Đại Học Y Hà Nội dạng tiếng Anh, tiếng Việt và tài liệu cầm tay. Kết quả: Tổng hợp trong 1678 nghiên cứu lọc tên bài và phần giới thiệu trên 3 trang cơ sở dữ liệu: Pubmed, Google Scholar, ESBCO host Research Databases được 987 nghiên cứu. Tiếp tục đánh giá chi tiết các tài liệu chọn được 16 tài liệu đạt tiêu chuẩn đưa vào phân tích: 12 nghiên cứu cho mục tiêu 1 và 4 nghiên cứu cho mục tiêu 2 . Kết quả phẩn tích gộp cho thấy: Độ lệch góc trung bình $1,4^{0}-6,53^{\circ}$, độ lệch cổ implant trung bình $0,46 \mathrm{~mm}-2,34 \mathrm{~mm}$, độ lệch chóp implant trung bình 0,67mm-2,53mm. Không có sự khác biệt đáng kể về mức độ tiêu xương rìa, cáćc biến chứng và tỷ lệ tồn tại của implant giữa phẫu thuật có sử dụng MDHPT và không sử dụng MHDPT. Kết luận: cấy ghép implant có sử dụng MDHPT và không sử dụng MHDPT đều mang lại kểt quả tương tự nhau về mức độ tiêu xương rìa, biến chứng và tỷ lệ tồn tại của implant. Tuy nhiên, việc sử dụng MHDPT có thể giúp implant được đặt vào có vị trí chính xác hơn theo cả 3 chiều trong xương hàm.

Tư khóa: implant nha khoa, máng hướng dẫn phẫu thuật, tổng quan

\section{SUMMARY}

\section{SURGICAL GUIDE TEMPLATE FOR DENTAL} IMPLANT: A SYSTEMATIC REVIEW

The important goal for successful implant placement is the ideal position of the implant in three

${ }^{1}$ Viện Đào tạo Răng hàm mặt-Trường Đại học Y Hà Nội ${ }^{2}$ Bểnh viện Răng hàm mặt Trung ương Hà Nội ${ }^{3}$ Trường Đai hoc Y Dược - Đại học Quốc Gia Hà Nộ Chịu trách nhiệm chính: Nguyễn Cao Thắng

Email: nctdentist@yahoo.com

Ngày nhận bài: 6.5.2021

Ngày phản biên khoa hoc: 2.7.2021

Ngày duyệt bài: 12.7.2021 dimensions in the bone, long-lasting, functional and aesthetic. The use of surgical guide template (SGT) in implant placement is believed to bring many benefits. Objectives: Describe and meta-analyse the accuracy of implant placement using SGT, marginal bone loss, complications and survival rate of implants with and without SGT. Materials and Methods: Systematic review and meta-analysis of data on accuracy of implant placement, marginal bone loss, complications and survival rate of implants using SGT and do not use SGT from articles, theses and theses on Pubmed, Google Scholar, EBSCOhost Research Databases, library of Hanoi Medical University in English, Vietnamese and hand-held documents. Results: A total of 987 eligible studies were selected from the literature search of 1678 titles from PubMed, Google Scholar, and EBSCO host Research Databases. Finally, 16 studies were included for meta-analysis: 12 studies for objective 1 and 4 studies for objective 2 . The results of the meta-analysis showed: angular deviation average $1.4^{0}-6.53^{\circ}$, average cervical deviation $0.46 \mathrm{~mm}-2.34 \mathrm{~mm}$, average apical deviation $0.67 \mathrm{~mm}$ $2.53 \mathrm{~mm}$. There were no significant differences in the degree of marginal bone loss, complications and implant survival between surgery with SGT and without SGT. Conclusion: Implants using SGT and without using SGT have similar results in terms of marginal bone loss, complications and implant survival rate. However, the use of SGT can help the implant to be placed in a more accurate position in all 3 dimensions in the bone.

Key words: dental implant, surgical guide implant, a review.

\section{I. ĐĂT VẤN ĐỀ}

Cây ghép Implant nha khoa là một trong những phương pháp phục hình mất răng ngày càng phổ biến do có nhiều đặc tính ưu việt so với các phương pháp phục hình răng truyền thống. Mục tiêu quan trọng để cấy ghép implant thành công là vị trí implant lý tưởng chính xác theo 3 chiêuu trong xương hàm, tồn tại lâu dài, đảm bảo được chức năng và thẩm mỹ.

Sự ra đời của chụp cắt lớp vi tính với chùm tia hình nón $(\mathrm{CBCT})$ với tính sẵn có ngày càng cao, bức xạ thấp, chi phí thấp, kế hoạch cấy ghép implant theo ba chiêu trước phẫu thuật đang trở nên phổ biến hơn. Phần mềm cho phép đặt implant ảo sử dụng dữ liệu kỹ thuật số thu được từ quá trình chụp $\mathrm{CBCT}$ và hình ảnh trong miệng bệnh nhân, chuyển vị trí implant đã lên kế hoạch trước phẫu thuật vào các MHDPT đã cải thiện kết quả điêu trị cấy ghép implant. 
Nhiều tác giả cho rằng, việc sử dụng MHDPT trong cấy ghép implant mang lại nhiều lợi ích, implant được đặt vào có vị trí chính xác hơn theo cả 3 chiều trong xương, hạn chế nhu cầu phẫu thuật mở vạt hoặc ghép xương dó đó là giảm chi phí, giảm sang chấn, nhanh liền thương [1].

Mặc dù kỹ thuật cấy ghép implant có sử dụng MHDPT được cho là có khả năng đạt được vị trí cây ghép chính xác hơn và ít xâm nhập hơn, nhưng kỹ thuật này cần phải được đánh giá nghiêm túc vì đã được thực hành lâm sàng trực tiếp trên bệnh nhân. Do đó, chúng tôi tiên hành nghiên cứu này với mục tiêu:

1. Mô tả độ chính xác của vi trí implant được cây ghép có sử dụng MHDPT trong các tài liệu đã công bố.

2. Mô tả mức độ tiêu xương rìa, các biến chứng và tỷ lệ tồn tại của implant được cây ghép có sử dụng MHDPT và không sử dụng MHDPT trong các tài liệu đã công bố.

\section{II. ĐỐI TƯỢNG VÀ PHƯƠNG PHÁP NGHIÊN CỨU}

2.1 Đối tượng: Các bài báo, luận văn, luận án trên trang cơ sở dữ liệu Pubmed, Google Scholar, EBSCOhost Research Databases, thu viện trường Đại Học $Y$ Hà Nội dạng tiếng Anh, tiểng Việt và tài liệu cầm tay.

\section{Tiêu chuẩn lựa chọn:}

* Theo tiêu chí PICO:

- Population/Patient (Đối tượng): Bệnh nhân mất răng được chỉ định cấy ghép implant.

- Intervention (can thiệp): Cây ghép implant nha khoa.

- Control/Comparison: So sánh vị trí implant thực tế sau cấy ghép có sử dụng MHDPT và vị trí trong kế hoạch, so sánh cây ghép implant có sử dụng MHDPT và không sử dụng MHDPT.

- Outcome: Vị trí implant, mức độ tiêu xương rìa, biến chứng cơ học và sinh học, tỷ lệ tồn tại của implant.

Tiêu chuẩn loại trừ: - Các bài báo không cung cấp đủ thông tin phục vụ cho nghiên cứu.

- Các bài dạng tổng quan.

- Các nghiên cứu trên động vật, tử thi.

\subsection{Phương pháp nghiên cứu:}

\section{Các bước tiến hành nghiên cứu:}

Bước 1: Xác định câu hỏi nghiên cứu: Phương pháp cấy ghép implant có sử dụng MHDPT có kết quả như thế nào, có ưu điểm gì so với phương pháp cấy ghép implant truyền thống không sử dụng MHDPT?

Bước 2: Xây dựng tiêu chuẩn lựa chọn/ loại trừ đối tượng.

Bước 3: Phương pháp, chiến lược tìm kiếm và nguồn dữ liệu

- Quá trình tìm kiếm được thực hiện từ 08/2020 đên 05/2021

- Từ khóa tìm kiếm online: dental implant, implant dentistry, surgical guide implant, surgical guide template for implant, computer-guided dental implant placement surgery

Bước 4: Lựa chọn nghiên cứu

Bước 5: Đánh giá chất lượng nghiên cứu

Sử dụng các công cụ đánh giá chất lượng nghiên cứu tùy thuộc vào loại thiết kế nghiên cứu:

- Công cụ CONSORT: nghiên cứu can thiệp

- Công cụ STROBE: nghiên cứu quan sát định lượng

Các nghiên cứu >17 điểm được lựa chọn vào nghiên cứu

Bước 6: Trích xuất dữ liệu, đo lường kết quả

Đo lường sự sai lệch giữa vị trí implant theo kế hoạch và vị trí implant thực tế: Trung bình và đô lệch chuẩn của các thông số sau được tính toán để đánh giá độ chính xác của vị trí cấy ghép (Hình 1):

a. Độ lệch góc (đo bằng độ): góc lệch giữa các trục dọc của implant theo kế hoạch và thực tế được đặt.

b. Độ lệch vai (đo bằng $\mathrm{mm}$ ): độ lệch tuyến tính giữa điểm vào (tức là trung tâm của platform) của các implant đã được lên kế hoạch và thực tế.

c. Độ lệch chóp (tính bằng $\mathrm{mm}$ ): độ lệch tuyến tính giữa điểm chóp của implant theo kễ hoach và thực tế được đăt.

d. Độ lệch độ sẩu (tính bằng $\mathrm{mm}$ ): độ lệch tuyến tính được đo theo hướng thẳng đứng giữa tâm platform của implant theo kế hoạch và thực tế được đặt.

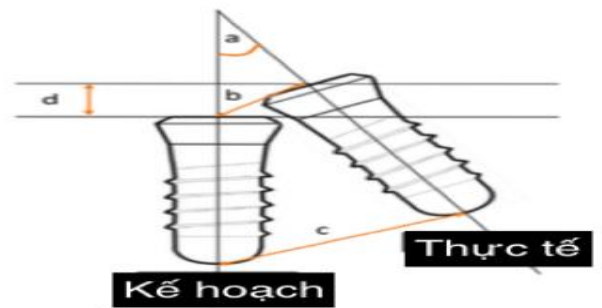

Hình 1: Thông số về độ chính xác của vị trí implant a: độ lệch góc; b: độ lệch vai; c: độ lệch chóp; d: độ lệch độ sâu

Mức độ tiều xương rìa, các biến chứng và tỷ lệ tồn tại của implant:

Đánh giá mức độ tiêu xương rìa xung quanh implant (tính bằng milimet) sao thời gian theo dõi của nghiên cứu.

Ghi nhận các biến chứng cơ học: mẻ sứ, bong mối trám composite dùng để trám lỗ phục hình 
trên implant, lỏng vít, lỏng phục hình,...

Ghi nhận các biến chứng sinh học: đau, sưng, chảy máu kéo dài, viêm quanh implant, áp xe,...

Đánh giá tỷ lệ tồn tại của implant sau thời gian theo dõi của các nghiên cứu.

Xử lý và phân tích số liệu: Số liệu được nhập và phân tích bằng phần mềm Comprehensive meta analysis V2.

\section{KẾT QUẢ NGHIÊN CỨU VÀ BÀN LUÂ̂N}

Tìm trên 3 trang cơ sở dữ liệu: Pubmed, Google Scholar, ESBCO host Research Databases được 1678 tài liệu tham khảo. Sau khi loại bỏ các

Bảng 1: Các nghiên cứu đủ tiêu chuẩn

\begin{tabular}{|c|c|c|c|c|c|c|}
\hline Tác giả & $\begin{array}{c}\text { Thiết kế } \\
\text { nghiên cứu }\end{array}$ & $\begin{array}{c}\text { Số lượng } \\
\text { bệnh nhấn }\end{array}$ & $\begin{array}{c}\text { Số lượng } \\
\text { implant }\end{array}$ & Tuổi & Vị trí implant & $\begin{array}{l}\text { Thời gian } \\
\text { theo dõi }\end{array}$ \\
\hline Fang Y. et al., 2019 & Tiến cứu & 32 & 40 & 56 & $\begin{array}{c}\text { Hàm trên + hàm } \\
\text { dưới }\end{array}$ & $\mathrm{NR}^{*}$ \\
\hline $\begin{array}{c}\text { Schelbert T. et al., } \\
2019\end{array}$ & Tiến cứu & 16 & 26 & $24-81$ & $\begin{array}{c}\text { Hàm trên + Hàm } \\
\text { dưới }\end{array}$ & $\mathrm{NR}^{*}$ \\
\hline $\begin{array}{l}\text { Cassetta M. et al., } \\
2014\end{array}$ & Hồi cứu & 20 & 225 & NR & $\begin{array}{c}\text { Hàm trên + Hàm } \\
\text { dưới }\end{array}$ & $\mathrm{NR}^{*}$ \\
\hline $\begin{array}{c}\text { D'haese J. et al., } \\
2012\end{array}$ & Tiến cứu & 13 & 78 & $\begin{array}{c}53,3 \\
(36-72)\end{array}$ & Hàm trên & 12 tháng \\
\hline $\begin{array}{l}\text { Di Giacomo G.A. et } \\
\text { al., } 2012\end{array}$ & Tiến cứu & 12 & 60 & $41-71$ & $\begin{array}{c}\text { Hàm trên + Hàm } \\
\text { dưới }\end{array}$ & 30 tháng \\
\hline $\begin{array}{c}\text { Pettersson A. et al., } \\
2012\end{array}$ & Hồi cứu & 11 & 139 & $\begin{array}{c}72 \\
(44-92)\end{array}$ & $\begin{array}{c}\text { Hàm trên + Hàm } \\
\text { dưới }\end{array}$ & 12 tháng \\
\hline $\begin{array}{l}\text { Stübinger S. et al., } \\
2014\end{array}$ & Tiến cứu & 10 & 44 & $\begin{array}{c}62,5 \\
(47-81)\end{array}$ & Hàm trên & 12 tháng \\
\hline $\begin{array}{l}\text { Vieira D.M. et al., } \\
2013\end{array}$ & Tiến cứu & 14 & 62 & NR & Hàm dưới & $\mathrm{NR}^{*}$ \\
\hline Ersoy AE et al., 2008 & Tiến cứu & 21 & 94 & NR & $\begin{array}{c}\text { Hàm trên + Hàm } \\
\text { dưới }\end{array}$ & $\mathrm{NR}^{*}$ \\
\hline $\begin{array}{l}\text { Smitkarn P et al., } \\
2019\end{array}$ & $\begin{array}{l}\text { Thử nghiệm lâm } \\
\text { sàng ngẫu nhiên } \\
\text { có đối chứng }\end{array}$ & 52 & 60 & NR & $\begin{array}{c}\text { Hàm trên + Hàm } \\
\text { dưới }\end{array}$ & $\mathrm{NR}^{*}$ \\
\hline $\begin{array}{c}\text { Magrin GL et al., } \\
2020\end{array}$ & $\begin{array}{l}\text { Thử nghiệm lâm } \\
\text { sàng ngấu nhiên } \\
\text { có đối chứng }\end{array}$ & 16 & 24 & 426,01 & Hàm dưới & $\mathrm{NR}^{*}$ \\
\hline Varga E et al., 2020 & $\begin{array}{l}\text { Thử nghiệm lâm } \\
\text { sàng ngẫu nhiên } \\
\text { có đối chứng }\end{array}$ & 101 & 207 & $\mid \begin{array}{c}42,11 \\
(18-75)\end{array}$ & $\begin{array}{c}\text { Hàm trên + Hàm } \\
\text { dưới }\end{array}$ & $\mathrm{NR}^{*}$ \\
\hline Pozzi A. et al., 2014 & $\begin{array}{l}\text { Thử nghieêm lâm } \\
\text { sàng ngấu nhiên } \\
\text { có đối chứng }\end{array}$ & 51 & 202 & 63,05 & $\begin{array}{c}\text { Hàm trên + Hàm } \\
\text { dưới }\end{array}$ & 1 năm \\
\hline $\begin{array}{l}\text { Amorfini L. et al., } \\
2017\end{array}$ & $\begin{array}{l}\text { Thử nghieêm lâm } \\
\text { sàng ngấu nhiên } \\
\text { có đối chứng }\end{array}$ & 24 & 70 & $\mid \begin{array}{c}55,7 \\
(39-75)\end{array}$ & Hàm trên & 2 năm \\
\hline $\begin{array}{l}\text { Tallarico M. et al., } \\
2018\end{array}$ & $\begin{array}{l}\text { Thử nghiệm lâm } \\
\text { sàng ngấu nhiên } \\
\text { có đối chứng }\end{array}$ & 20 & 62 & 64,05 & $\begin{array}{c}\text { Hàm trên + Hàm } \\
\text { dưới }\end{array}$ & 5 năm \\
\hline $\begin{array}{l}\text { Vercruyssen M. et } \\
\text { al., } 2014\end{array}$ & $\begin{array}{l}\text { Thử nghiệm lâm } \\
\text { sàng ngấu nhiên } \\
\text { có đối chứng }\end{array}$ & 59 & 263 & 58 & $\begin{array}{c}\text { Hàm trên + hàm } \\
\text { dưới }\end{array}$ & 2 năm \\
\hline
\end{tabular}
đánh giá chi tiết tiêu đề và tóm tắt của các tài liệu, có 38 tài liệu đủ điều kiện đọc toàn văn. Sau khi đọc toàn bộ tài liệu, chọn được 16 tài liêu đạt tiêu chuẩn đưa vào phân tích: 12 nghiên cứu cho mục tiêu 1 và 4 nghiên cứu cho mục tiêu 2 .

3.1 Đặc điểm nghiên cứu. Đặc điểm của các bài báo được đưa vào tổng quan này được trình bày trong bảng 1 . Tổng số 472 bệnh nhẩn và 1656 implant được phân tích trong 7 thử nghiệm lâm sàng ngẫu nhiên có đối chứng, 7 nghiển cứu tiến cứu và 2 nghiên cứu hồi cứu. tài liệu trùng lặp còn 987 nghiên cứu. Tiếp tục 
3.2. Độ chính xác của vị trí implant được cấy ghép có sử dụng máng hướng dẩn phầu thuật trong các tài liệu đã công bố. Độ lệch góc, độ lệch cổ và độ lệch chóp implant thực tế đã cấy so với vị trí trong kế hoạch đã được mô tả trong tất cả các nghiên cứu trong tổng quan, được trình bày dưới dạng giá trị trung bình và độ lệch chuẩn $(\mathrm{SD})$ của các phép đo (Bảng 2). Độ lệch góc trung bình giữa các nghiên cứu nằm trong khoảng từ $1,4^{0}$ (tối thiểu) đến
6,530 (tối đa) [2],[3]. Độ lệch cổ implant trung bình là $0,46 \mathrm{~mm}$ đến $2,34 \mathrm{~mm}$ [2],[1]. Độ lệch chóp implant trung bình là $0,67 \mathrm{~mm}$ đên 2,53 $\mathrm{mm}[2],[1]$. Implant có độ lệch giữa thực tế và kễ hoạch lớn hơn chủ yếu ở hàm trên hoặc ở những khoảng mất răng không có giới hạn xa, tuy nhiên sự khác biệt là không có ý nghĩa thống kê. Sai số độ sâu implant trung bình là $0,15 \mathrm{~mm}$ đến $0,65 \mathrm{~mm}$, tuy nhiên nhiều nghiên cứu không báo cáo dữ liệu này (Bảng 2) [4].

Bảng 2: Dữ liệu về độ chính xác của implant được cây ghép có sử dụng máng hướng dẫn phẫu thuật

\begin{tabular}{|c|c|c|c|c|}
\hline \multicolumn{3}{|l|}{ dân phâu thuật } & \multicolumn{2}{|r|}{ (NR*: không báo cáo) } \\
\hline Tác giả & $\begin{array}{l}\text { Trung bình độ } \\
\text { lệch góc }\left({ }^{0}\right)\end{array}$ & $\begin{array}{l}\text { Trung bình độ } \\
\text { lệch cổ (mm) }\end{array}$ & \begin{tabular}{|c|}
$\begin{array}{c}\text { Trung bình độ } \\
\text { lệch chóp } \\
(\mathbf{m m})\end{array}$ \\
\end{tabular} & $\begin{array}{c}\text { Trung bình độ } \\
\text { lệch chiều sâu } \\
\text { của implant (mm) }\end{array}$ \\
\hline al., 2019 & $1,40(0,30-2,57)$ & $0,46(0-1,15)$ & $0,67(0,14-1,19)$ & $0,15(0,10-0,82)$ \\
\hline Schelbert T. et al., 2019 & $4,11 \pm 0,52$ & 0,11 & $1,22 \pm 0,11$ & $0,65 \pm 0,11$ \\
\hline Cassetta M. et al., 2014 & $4,67 \pm 2,68$ &, 60 & 2,19 & $\mathrm{NR}^{*}$ \\
\hline D'haese J. et al., 2012 & $2,6 \pm 1,61$ & $0,91 \pm 0,44$ & $1,13 \pm 0,52$ & $\mathrm{NR}^{*}$ \\
\hline Di Giacomo G.A. et al., 2012 & & $1,35 \pm 0,65$ & $1,79 \pm 1,01$ & $\mathrm{NR}^{*}$ \\
\hline Pettersson A. et al., 2012 & $6+2$ & 72 & 1,01 & 0,15 \\
\hline Stübinger S. et al., 2014 & $2,39 \pm 0,97$ & $0,71 \pm 0,40$ & $0,77 \pm 0,38$ & $0,47 \pm 0,496$ \\
\hline Vieira D.M. et al., 2013 & $1,90 \pm 0,57$ & $1,88 \pm 0,78$ & $2,35 \pm 1,43$ & $\mathrm{NR}^{*}$ \\
\hline Ersoy AE & & & & $\mathrm{NR}^{*}$ \\
\hline Smitkarn & & & & $\mathrm{NR}^{*}$ \\
\hline Magrin GL et al., 2020 & 1,1 & $2,34 \pm 1,01$ & $2,53 \pm 1,11$ & $\mathrm{NR}^{*}$ \\
\hline Varga E et al., 2020 & $3,04 \pm 1,51$ & $1,40 \pm 0,54$ & $1,59 \pm 0,59$ & $\mathrm{NR}^{*}$ \\
\hline
\end{tabular}

Tổng quan này đã đánh giá các tài liệu liên quan đến độ chính xác của implant được cấy ghép có sử dụng MHDPT.

Các tác giả đã thảo luận về các biến số có khả năng gây ra sai lệch giữa vị trí implant dự kiến và vị trí implant thực tế được cấy ghép có sử dụng MHDPT. Có thể nhấn mạnh rằng trong quá trình thu nhận, xử lý và thao tác hình ảnh, các sai số hoàn toàn có thể xảy ra. Các lỗi cũng có thể xảy ra trong quá trình sản xuất MHDPT trong giai đoạn mô phỏng phẫu thuật trên phần mềm, về độ chính xác của máy tạo mẫu, trong các đặc tính của vật liệu được sử dụng, ở sự phù hợp giữa các trụ của hướng dẫn, mũi khoan của hệ thống cấy ghép. Các lỗi sản xuất có thể có tác động tích lũy, có thể tạo ra các kết quả lâm sàng không thuận lợi. Việc lựa chọn mô nâng đõ̃ MHDPT cũng góp phần làm thay đổi vị trí implant thực tế so với implant trong kế hoạch ban đầu.

Để giảm thiểu sự thiếu chính xác có thể xảy ra trong cấy ghép implant có sử dụng MHDPT, D’haese và cộng sự đã sử dụng các thanh được hỗ trợ bằng niêm mạc, điều chỉnh thích hợp với bề mặt niêm mạc và được gắn chặt bằng vít, lưu ý rằng kết quả trung bình của độ lệch (góc: $2,6^{\circ}$, cổ implant: $0,91 \mathrm{~mm}$ và chóp implant: $1,13 \mathrm{~mm}$ ) thấp hơn so với kết quả được báo cáo bởi các nghiên cứu trước đây.

3.3. Mức độ tiêu xương rìa, các biến chứng và tỷ lệ tôn tại của implant được cây ghép có sử dụng máng hướng dấn phẫu thuật (CG) và không sử dụng máng hướng dẫn phẫu thuật $(\mathrm{FH})$ trong các tài liệu đã công bố

Tiêu xương rìa: Có 4 nghiên cứu đánh giá mức độ tiêu xương rìa quanh implant (tính bằng milimét). Phân tích tổng thể về sự khác biệt trung bình cho thây không có sự khác biệt đáng kể về mức độ tiêu xương rìa giữa phẫu thuật có máng hướng dẫn và phẫu thuật tự do.

Biến chứng cơ học: Ba nghiên cứu báo cáo có các biến chứng cơ học. Các biến chứng thường gặp nhất là mẻ sứ hoặc bong composite dùng để trám dính phục hình ( $\mathrm{CG}=5, \mathrm{FH}=2)$ và lỏng vít $(\mathrm{CG}=1, \mathrm{FH}=4)$. Các biến chứng khác là gãy phục hình tạm $(\mathrm{CG}=3, \mathrm{FH}=3)$ và phục hình thất baii $(C G=0, F H=1)$. Không có sự khác biệt giữa kỹ thuật cấy ghép implant có sử dụng máng hướng dẫn phẫu thuật và không sử dụng máng hướng dẫn phẫu thuật về các biến chứng cơ học.

Biến chứng sinh học: Ba nghiên cứu báo cáo các biến chứng sinh học. Các biến chứng thường 
gặp nhất là đau ( $\mathrm{CG}=4, \mathrm{FH}=2)$ và viêm quanh implant $(\mathrm{CG}=3, \mathrm{FH}=1)$. Một biến chứng khác là áp xe $(\mathrm{CG}=2, \mathrm{FH}=0)$. Khồng có sự khác biệt giữa hai kỹ thuật về các biến chứng sinh học.

Tỷ lê tồn tai của implant: Tất cả các nghiên cứu đều báo cáo số lượng implant thất bại. Tỷ lệ tồn tại của implant là $99,7 \%$ đối với phẫu thuât có hướng dẫn bằng máy tính và $98,6 \%$ đối với phẫu thuật tự do. Phân tích tổng hợp cho thấy không có sự khác biệt đáng kể về tỷ lệ tồn tại giữa hai kỹ thuật phẫu thuật.

Bảng 3: Dữ liệu về mức độ tiêu xương ria, các biến chứng và tỷ lên tồn tại của implant được cây ghép có sử dụng máng hướng dẫn phẫu thuật và không sử dụng máng hướng dẫn phẩu thuật

\begin{tabular}{|c|c|c|c|c|c|}
\hline Tác giả & Phương pháp & $\begin{array}{c}\text { Mức độ tiêu } \\
\text { xương rìa } \\
\text { (mm) }\end{array}$ & $\begin{array}{l}\text { Biến chứng } \\
\text { cơ học }\end{array}$ & $\begin{array}{l}\text { Biến chứng } \\
\text { sinh học }\end{array}$ & $\begin{array}{c}\begin{array}{c}\text { Tỷ lệ tôn tại } \\
\text { của împlant } \\
(\%)\end{array} \\
\end{array}$ \\
\hline \multirow{2}{*}{$\begin{array}{l}\text { Amorfini L. } \\
\text { et al., } 2017 \\
{[5]}\end{array}$} & $\begin{array}{c}\text { Có sử dung máng hướng } \\
\text { dẫn phấu thuật }\end{array}$ & $1,95 \pm 0,7$ & $\begin{array}{l}1 \text { gãy phục } \\
\text { hình tạm }\end{array}$ & & 97,2 \\
\hline & $\begin{array}{l}\text { Không sử dưng máng } \\
\text { hướng dẩn phẫu thuật }\end{array}$ & $1,93 \pm 0,4$ & $\begin{array}{l}3 \text { gãy phục } \\
\text { hình tạm }\end{array}$ & & 100 \\
\hline \multirow{2}{*}{$\begin{array}{c}\text { Tallarico M. } \\
\text { et al., } 2018 \\
\text { [6] }\end{array}$} & $\begin{array}{c}\text { Có sử dunng máng hướng } \\
\text { dẫn phẫu thuật }\end{array}$ & $0,87 \pm 0,40$ & $\begin{array}{c}4 \\
1 \text { gãy phục } \\
\text { hinh tạm } \\
3 \text { vơ sứ }\end{array}$ & 2 (đau) & 100 \\
\hline & $\begin{array}{l}\text { Không sử dụng máng } \\
\text { hướng dẫn phâuu thuậtt }\end{array}$ & $1,29 \pm 0,31$ & $\begin{array}{c}3 \\
2 \text { lỏng vít } \\
1 \text { võ sứ }\end{array}$ & \begin{tabular}{|c|}
2 \\
1 đau \\
1 viêm quanh \\
implant với tiêu \\
xương $3,5 \mathrm{~mm}$ \\
\end{tabular} & 93,33 \\
\hline \multirow{2}{*}{$\begin{array}{l}\text { Pozzi A. et } \\
\text { al., 2014 } \\
\text { [7] }\end{array}$} & $\begin{array}{c}\text { Có sử dựng máng hướng } \\
\text { dấn phẫu thuật }\end{array}$ & $0,71 \pm 0,25$ & $\begin{array}{c}4 \\
1 \text { gãy phục } \\
\text { hình tạm } \\
2 \text { võ sứ } \\
1 \text { lỏng vít } \\
\end{array}$ & 2 (đau) & 100 \\
\hline & $\begin{array}{l}\text { Không sử dụng máng } \\
\text { hướng dẫn phâuu thuậtt }\end{array}$ & $0,80 \pm 0,29$ & $\begin{array}{c}4 \\
1 \text { thất bai } \\
1 \text { võ sứ } \\
2 \text { lỏng vít }\end{array}$ & $\begin{array}{l}1 \text { (đau và } \\
\text { sưng) }\end{array}$ & 98,9 \\
\hline \multirow{2}{*}{$\begin{array}{l}\text { Vercruyssen } \\
\text { M. et al., } \\
2014 \text { [8] }\end{array}$} & $\begin{array}{c}\text { Có sử dựng máng hướng } \\
\text { dấn phẫu thuật }\end{array}$ & $0,04 \pm 0,34$ & & \begin{tabular}{|c|}
5 \\
$\begin{array}{c}3 \text { viêm quanh } \\
\text { implant } \\
2 \text { áp xe }\end{array}$ \\
\end{tabular} & 100 \\
\hline & $\begin{array}{l}\text { Không sử dung máng } \\
\text { hướng dẩn phẩu thuậtt }\end{array}$ & $0,02 \pm 0,27$ & & 0 & 100 \\
\hline
\end{tabular}

Tổng quan này bao gồm các nghiên cứu so sánh kỹ thuật cấy ghép implant có sử dụng MHDPT và kỹ thuật phâu thuật tự do để đă̆t implant về mức độ tiêu xương rìa, các biến chứng cơ học và sinh học, tỷ lệ tồn tại của implant. Kết quả thu được từ phân tích tổng hợp đã xác minh rằng các kỹ thuật cấy ghép implant có sử dụng MHDPT và không sử dụng MHDPT cho tỷ lệ tiêu xương rìa, biến chứng cơ học, biến chứng sinh học và tỷ lệ tồn tại của implant tương tự nhau.

Cấy ghép implant có sử dụng MHDPT thể hiện một lợi thế lớn liên quan đến độ chính xác của vị trí implant, cho phép thực hiện phục hình tạm sớm và cần ít thời gian hơn để thích ứng với phục hình tạm trong các quy trình tải lực tức thì.
Tuy nhiên, việc cấy ghép implant có sử dụng MHDPT có nhược điểm là chi phí cao hơn.

Một số nghiên cứu thực nghiệm đã báo cáo rằng cấy ghép implant có sử dụng MHDPT không cho phép dung dịch làm mát tiếp xúc trực tiếp với mũi khoan trong quá trình chuẩn bị xương, điều này có thể dẫn đến nhiệt độ xương tăng lện và có thể cản trở quá trình liền xương. Phẫu thuật tự do, không sử dụng MHDPT, cho phép tiếp xúc nhiều hơn của dung dịch làm mát trên mũi khoan, giảm nhiệt độ trong quá trình khoan xương, và ngăn ngừa quá nhiệt và tiêu xương. Tuy nhiên, hiệu ứng này không được quan sát thấy trong nghiên cứu hiện tại, vì không có sự khác biệt giữa cấy ghép implant có sử dụng 
MHDPT và không sử dụng MHDPT liên quan đến mức độ tiêu xương rìa hoặc tỷ lệ tồn tại của implant, là những kết quả liên quan đên sự tích hợp xương. Rõ ràng rằng vị trí chính xác của implant là kết quả của việc lập kế hoạch chính xác bằng cách sử dụng phần mềm nha khoa và sự phù hợp của MHDPT với các răng hoặc niêm mạc còn lại của bệnh nhân. Điều này phụ thuộc rất lớn vào khả năng của kỹ thuật viên phòng thí nghiệm và máy CAM.

Có thể kết luận rằng cả phẫu thuật có hướng dẫn và phẫu thuật tự do đều mang lại kết quả tương tự nhau về $\mathrm{MB} L$, biến chứng và tỷ lệ tồn tại của implant. Tuy nhiên, do những hạn chế của nghiên cứu này, nên nghiên cứu thểm cần được thực hiện để nâng cao hiểu biết của chúng ta về chủ đề này.

\section{KẾT LUÂ̂N}

Có thể kêt luân rằng cấy ghép implant có sử dụng MDHPT và không sử dụng MHDPT đều mang lại kết quả tương tự nhau về mức độ tiêu xương rìa, biến chứng cơ học, biến chứng sinh học và tỷ lệ tồn tại của implant. Tuy nhiên, việc sứ dụng MHDPT có thể giúp implant được đặt vào có vị trí chính xác hơn theo cả 3 chiêu trong xương với các cải tiến kỹ thuật hoặc sử dụng các bộ phận cố định MDHPT trong quá trình cây ghép implant.

\section{TÀI LIỆU THAM KHẢO}

1. Magrin GL, Rafael SNF, Passoni BB, Magini RS, Benfatti CAM, Gruber R, et al. Clinical and tomographic comparison of dental implants placed by guided virtual surgery versus conventional technique: A split-mouth randomized clinical trial. J
Clin

Periodontol.

(https://doi.org/10.1111/jcpe.13211).

2. Fang $Y$, An $X$, Jeong S.M, Choi B.H. Accuracy of computer-guided implant placement in anterior regions. J Prosthet Dent 2019;121(5):836-842 (https://doi.org/10.1016/j.prosdent.2018.07.015).

3. Di Giacomo GA, da Silva JV, da Silva AM, Paschoal GH, Cury PR, Szarf G, et al. Accuracy and complications of computer-designed 24. selective laser sintering surgical guides for flapless dental implant placement and immediate definitive prosthesis installation. J Periodontol 2012;83:410-9 (https://doi.org/10.1902/jop.2011.110115).

4. Schelbert $T$, Gander $T$, Blumer $M$, et al. Accuracy of Computer-Guided Template-Based Implant Surgery: A Computed Tomography-Based Clinical Follow-Up Study. Implant Dent 2019;28(6):556-563

(https://doi.org/10.1097/ID.0000000000000936).

5. Amorfini L, Migliorati M, Drago S, SilvestriniBiavati A. Immediately loaded implants in rehabilitation of the maxilla: $a$ two-year randomized clinical trial of guided surgery versus standard procedure. Clin Implant Dent Relat Res 2017;19:280-(https://doi.org/10.1111/cid.12459).

6.Tallarico M, Esposito M, Xhanari E, Caneva M Meloni SM. Computer-guided vs freehand placement of immediately loaded dental implants: 5-year postloading results of a randomised controlled trial. Eur J Oral Implantol 2018;11:203-13.

7. Pozzi A, Tallarico M, Marchetti M, Scarfo B Esposito M. Computer-guided versus free- hand placement of immediately loaded dental implants: 1-year post-loading results of a multicentre randomised controlled trial. Eur J Oral Implantol 2014;7:229-42.

8.Vercruyssen $M$, van de Wiele $G$, Teughels $\mathbf{W}$, Naert I, Jacobs R, Quirynen M. Implant- and patient-centred outcomes of guided surgery, a 1year follow-up: an RCT comparing guided surgery with conventional implant placement. J Clin Periodontol 2014;41:1154-60 (https://doi.org/10.1111/jcpe.12305).

\section{ĐẶC ĐIỂM PHÂN BỐ RĂNG VĨNH VIỄN NGÂM Ở BÊ̂NH NHÂN NẮN CHİNH RĂNG}

\section{TÓM TẮT}

Nghiên cứu mô tả cắt ngang trên phim X-quang của 908 bệnh nhân đến khám và điều trị nắn chỉnh răng với mục tiêu: Mô tả đặc điểm phân bố răng vĩnh

\footnotetext{
${ }^{1}$ Viện Đào Tạo Răng Hàm Mặt, Đại học Y Hà Nộ 2 Bềnh viên Răng Hàm Mặt Trung Ương Hà Nội Chịu trách nhiệm chính: Biện Thị Nhàn Email: biennhan14295@gmail.com Ngày nhận bài: 11.5.2021

Ngày phản biên khoa học: 2.7.2021

Ngày duyệt bài: 15.7.2021
}

Biện Thị Nhàn ${ }^{1}$, Nguyễn Thị Bích Ngọc ${ }^{1}$, Nguyễn Thanh Huyền ${ }^{2}$, Đào Thị Hằng Nga ${ }^{1}$ viễn ngầm (trừ răng hàm lớn thứ ba) ở nhóm đối tượng này. Kết quả: Tỷ lê bênh nhân có răng vĩnh viển ngầm (trừ răng hàm lởn thứ ba) trên bênh nhân nắn chỉnh răng là $8,59 \%$, nam cao hơn nữ $(p<0,05)$. Tuổi nhỏ nhất có răng ngầm là 6 . Răng ngầm chủ yếu gặp ở hàm trên. Những răng ngầm hay gặp nhất là răng nanh hàm trên $(4,52 \%)$ và răng cửa giữa hàm trên $(3,52 \%)$. Đa số các trường hợp có 1 răng ngầm. Kết luấn: Kết quả của nghiên cứu đưa ra cái nhìn tổng quát về phân bố răng ngầm ở cả hai hàm, gợi ý về tuổi xuất hiện răng ngầm, khả năng ngầm của răng, vùng răng, cung cấp cơ sở cho việc tăng cường theo dõi và đưa ra các biện pháp can thiệp dự phòng 